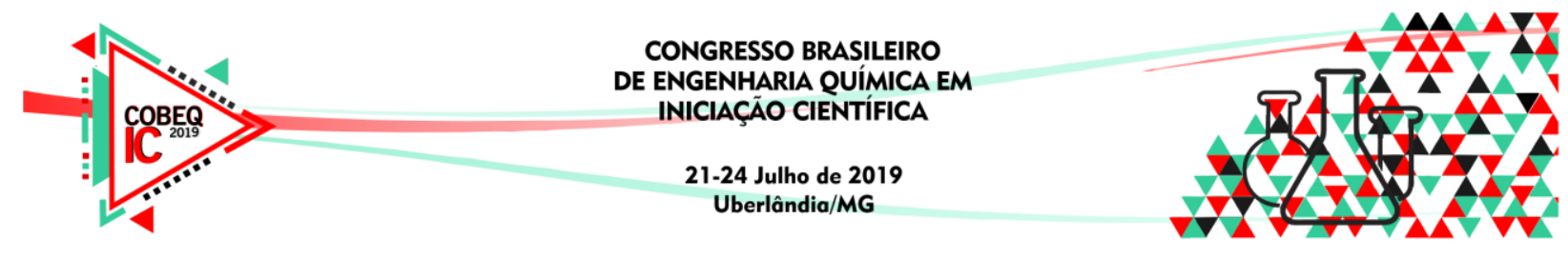

\title{
DESENVOLVIMENTO DO PROGRAMA DE COMPUTADOR "MMQ" DE REGRESSÃO LINEAR MÚLTIPLA NA CALCULADORA HP PRIME E SUA APLICAÇÃO NA DISCIPLINA DE CINÉTICA HETEROGÊNEA
}

\author{
M. F. PINHEIRO ${ }^{1}$, F. Á. RODRIGUES ${ }^{2}$ \\ ${ }^{1}$ Universidade Federal de Viçosa, Departamento de Química \\ ${ }^{2}$ Universidade Federal de Viçosa, Departamento de Química \\ E-mail para contato: matheusfpinheiro@gmail.com
}

\begin{abstract}
RESUMO - Os catalisadores estão cada vez mais presentes nos processos químicos industriais, visando uma maior eficiência dos mesmos. $\mathrm{O}$ estudo das mudanças reacionais que os catalisadores trazem, incluindo os novos mecanismos, são de grande importância no entendimento do processo, visando uma implementação industrial. $O$ presente trabalho apresenta o programa de computador "MMQ", criado para a calculadora HP Prime, buscando aplicar o método numérico dos "Mínimos Múltiplos Quadrados" para as regressões lineares múltiplas, na dedução de mecanismos de reações catalíticas heterogêneas.
\end{abstract}

\section{INTRODUÇÃO}

Os catalisadores são substâncias que interferem na velocidade de uma reação, através de alterações no caminho da mesma, e saem inalteradas ao fim do processo. Essas substâncias foram utilizadas durante séculos na fabricação de alimentos, mas apenas no século XIX os estudos sobre catalisadores iniciaram uma sistematização. Hoje, a utilização de catalisadores é necessidade na indústria, sendo partícipes em processos básicos ou complexos, como na indústria petroquímica (FOGLER, 2009). Segundo Maia (2010), a catálise heterogênea possui 7 etapas, das quais são 4 físicas e 3 químicas. De acordo com Fogler (2009), nas reações catalíticas heterogêneas em que as etapas químicas são as limitantes, pode-se deduzir o mecanismo das mesmas através da análise de dados de um reator diferencial.

A dedução do mecanismo é realizada através de uma análise qualitativa inicial dos dados, e em seguida uma aplicação do Modelo de Langmuir-Hinshelwood, que consiste numa sequência de etapas, dependendo da quantidade de sítios ativos envolvidos na reação, e do tipo de adsorção das substâncias, associativa ou dissociativa (SCHMAL, 2010). Segundo Fogler (2009), a compatibilidade do mecanismo deduzido e a descoberta das constantes de velocidade de reação são respostas que podem ser obtidas com uma regressão, esta pode ser linear simples ou não linear. As regressões lineares simples são solucionadas pela maioria das calculadoras científicas. Já as regressões não lineares são realizadas por programas de computador mais complexos como o Polymath ou Matlab. Uma forma alternativa à regressão não linear é a linearização do modelo, através de manipulações algébricas, e a realização de uma regressão linear múltipla, através do método numérico dos Mínimos Múltiplos 


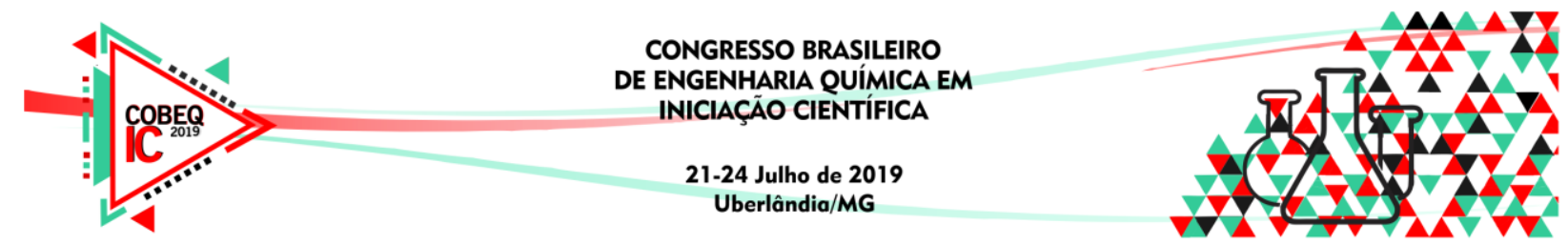

Quadrados, fornecendo resultados estimados para as constantes de velocidade de reação e sobre a coerência das deduções, de uma forma mais simplificada (FOGLER, 2009).

A partir da motivação citada, o trabalho a seguir baseia-se no desenvolvimento do programa de computador "MMQ", programado na linguagem HP PPL e aplicável na calculadora HP PRIME. O programa foi construído a partir do método numérico de regressão linear múltipla dos Mínimos Múltiplos Quadrados, direcionado para a resolução de problemas de dedução da lei de velocidade de reações heterogêneas, possuintes as etapas químicas como limitantes da reação.

\section{MATERIAIS E MÉTODOS}

\subsection{Regressão Linear Múltipla - Método dos Mínimos Quadrados}

A regressão linear múltipla, pelo método dos mínimos quadrados, gera uma função que busca retratar um fenômeno que possui mais de uma variável independente envolvida. Através da Equação 1 pode-se representar o caso matematicamente. Onde $f\left(x_{1}\right), \ldots, f\left(x_{2}\right)$ são $p$ funções lineares de $x_{1}, \ldots, x_{p}$, sendo representadas como na Equação 2. Somando todas as constantes $\alpha 0$ em um termo $\beta$, e unindo todas em uma $f(x)$, chega-se na Equação 3 .

$$
\begin{gathered}
y=f\left(x_{1}, \ldots, x_{P}\right)=f\left(x_{1}\right)+\cdots+f\left(x_{p}\right), p \geq 2 \\
f\left(x_{1}\right)=\alpha 0_{1}+\alpha_{1} x_{1}, \ldots, f\left(x_{p}\right)=\alpha 0_{p}+\alpha_{p} x_{p} \\
f(x)=\sum_{i=1}^{p} f\left(x_{p}\right)=\beta+\alpha_{1} x_{1}, \ldots, \alpha_{p} x_{p} ; p \geq 2
\end{gathered}
$$

Para o cálculo do erro gerado, utiliza-se o desvio quadrático $S$ oriundo da soma dos desvios quadráticos de cada ponto, representado na Equação 4. Inserindo as representações de cada $f(x)$ apresenta-se a Equação 4.

$$
S=S\left(\beta, \alpha_{1}, \ldots, \alpha_{p}\right)=\sum_{i=1}^{n}\left(y_{i}-f\left(x_{1 i}, \ldots, x_{p i}\right)\right)^{2}
$$

Aplicando o Teorema de Máximos e Mínimos do cálculo na função $S$, busca-se um ponto crítico de mínimo para o erro. Para isto, iguala-se as derivadas parciais a zero, como na Equação 5.

$$
\frac{\partial S\left(\beta, \alpha_{1, \ldots,} \alpha_{p}\right)}{\partial \beta}=\frac{\partial S\left(\beta, \alpha_{1, \ldots,} \alpha_{p}\right)}{\partial \alpha_{1}}=\frac{\partial S\left(\beta, \alpha_{1, \cdots,} \alpha_{p}\right)}{\partial \alpha_{p}}=0
$$

$\mathrm{O}$ resultado da derivação é um sistema linear de $p+1$ equações e com $p+1$ incógnitas. Ao resolver esse sistema, obtém-se a função de ajuste da regressão. A Equação 6 representa o sistema em forma matricial, representada simplificadamente na Equação 7:

$$
\left[\begin{array}{cccc}
n & \sum x_{l i} & \ldots & \sum x_{p i} \\
\sum x_{l i} & \sum x_{l i} x_{l i} & \ldots & \sum x_{l i} x_{p 1} \\
\vdots & \vdots & \ddots & \vdots \\
\sum x_{p 1} & \sum x_{l 1} x_{p 1} & \ldots & \sum x_{p 1} x_{p 1}
\end{array}\right]\left[\begin{array}{c}
\beta \\
\alpha_{1} \\
\vdots \\
\alpha_{p}
\end{array}\right]=\left[\begin{array}{c}
\sum y_{i} \\
\sum x_{l i} y_{i} \\
\vdots \\
\sum x_{p i} y_{i}
\end{array}\right]
$$




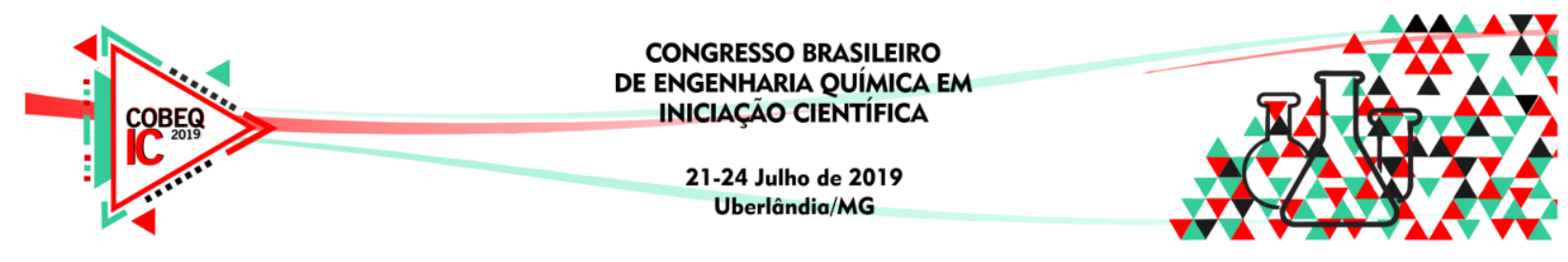

$[$ Matriz X] x [Matriz K] $=[$ Matriz Y $]$

Para obter o valor das constantes $\beta, \alpha_{1}, \ldots, \alpha_{p}$ basta encontrar a inversa da Matriz X e multiplicá-la pela Matriz Y.

\subsection{Modelo de Langmuir-Hinshelwood}

A explanação sobre o Modelo de Langmuir-Hinshelwood, por motivos didáticos, foi feita a partir do exemplo de reação irreversível que acontece em três etapas: a) ocorre a adsorção do reagente representado na Equação 8 sendo que A é a molécula livre no interior do poro catalisador, s é o sítio ativo na superfície, A-s a molécula adsorvida sobre a superfície, Ka e Ka' as constantes de adsorção e dessorção de A; b) após a adsorção, ocorre a reação química representada na Equação 9, onde K é a velocidade específica da reação; c) por fim tem-se a dessorção do produto reacional, vide Equação 10. Desprezando efeitos difusivos no sistema, a reação química é considerada a etapa limitante.

$$
\begin{aligned}
& A+s \underset{K a^{r}}{\stackrel{K a}{\longrightarrow}} A-s \\
& A-s \stackrel{K}{\longrightarrow} R-s \\
& R-s \underset{K d^{\prime}}{\stackrel{K d}{\longrightarrow}} R+s
\end{aligned}
$$

Admitindo-se que a reação química é irreversível, de primeira ordem e limitante do processo, tem-se a Equação 11 como representante da taxa, sendo $\theta_{A}$ a fração de sítios ativos ocupados pela espécie A. Sabendo que A e R estão adsorvidos nos sítios ativos, tem-se o mecanismo representado na Equação 12:

$$
\begin{aligned}
& r=k \theta_{A} \\
& \left(-r_{A}\right)=\frac{k K_{A} P_{A}}{\left(1+K_{A} P_{A}+K_{R} P_{R}\right)}
\end{aligned}
$$

\subsection{Programa de Computador MMQ}

A calculadora utilizada no projeto desenvolvido foi a HP Prime, que possui utilidades avançadas, porém com um uso bastante intuitivo. O programa de computador desenvolvido foi programado na linguagem HP PPL, que é baseada na popular linguagem Pascal e possui um sistema de sintaxe relativamente simples, entretanto contém uma gama interessante de possibilidades de programação.

O programa é baseado em quatro etapas, sendo as três primeiras de entrada de dados, e a última com o resultado da regressão linear múltipla. Ao iniciar o primeiro ambiente, o controlador deve inserir o número de experimentos registrados. Em seguida, o controlador é direcionado ao segundo ambiente, onde o programa pede o número de variáveis 


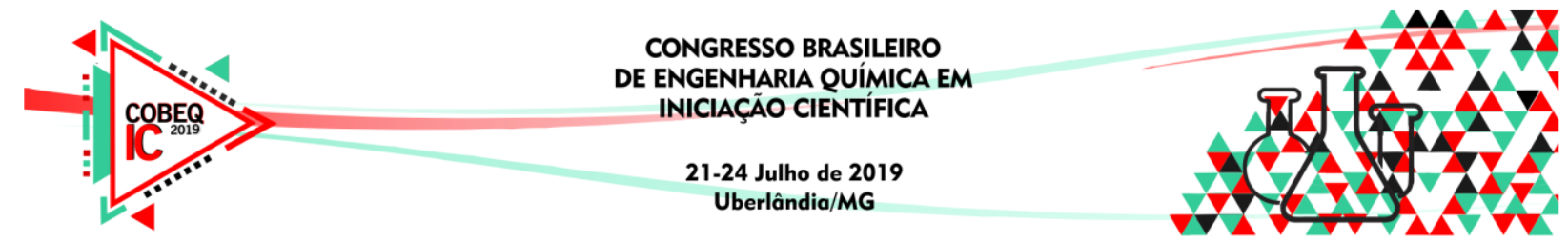

independentes, ou X(s) da equação linearizada. No terceiro ambiente, o programa exibe uma matriz ou uma tabela de entrada de dados. Nesta tabela, a primeira coluna deve ser preenchida com os dados referentes aos Ys das funções, ou as variáveis dependentes. As colunas vizinhas devem ser preenchidas com os dados instantâneos das variáveis dependentes. O programa recebe os dados inseridos nos três ambientes, realiza a regressão linear múltipla, e exibe os resultados no quarto ambiente.

\subsection{Estudo de caso}

O método numérico dos mínimos múltiplos quadrados é bastante confiável como aproximação em casos onde, inicialmente, seria necessária uma regressão diferencial não linear para encontrar constantes. Buscando explicitar o bom funcionamento da ferramenta desenvolvida e a faixa de erro na aproximação utilizada, um problema de caso que envolve aplicação do programa MMQ em cinética heterogênea na dedução do mecanismo reacional, foi resolvido com o programa em questão, e com a ferramenta Polymath, proporcionando uma comparação ao fim das resoluções.

\section{RESULTADOS E DISCUSSÕES}

A Tabela 1 apresenta resultados experimentais obtidos em um reator diferencial referentes à reação catalítica irreversível em fase gasosa expressa na Equação 13.

$$
A+B \rightarrow C
$$

Tabela 1 - Resultados experimentais

\begin{tabular}{|c|c|c|c|c|}
\hline Experimento & $\mathrm{P}_{\mathrm{A}}(\mathrm{atm})$ & $\mathrm{P}_{\mathrm{B}}(\mathrm{atm})$ & $\mathrm{P}_{\mathrm{C}}(\mathrm{atm})$ & $\begin{array}{c}\mathrm{r} .10^{3} \\
(\mathrm{~mol} / \text { (min.gcat) })\end{array}$ \\
\hline \hline 1 & 0,1 & 1 & 2 & 0,073 \\
\hline 2 & 1 & 10 & 2 & 3,42 \\
\hline 3 & 10 & 1 & 2 & 0,54 \\
\hline 4 & 1 & 20 & 2 & 6,80 \\
\hline 5 & 1 & 20 & 10 & 2,88 \\
\hline 6 & 20 & 1 & 2 & 0,56 \\
\hline 7 & 1 & 1 & 2 & 0,34 \\
\hline 8 & 1 & 20 & 5 & 4,5 \\
\hline
\end{tabular}

Baseado nos dados da tabela acima, deve-se propor um mecanismo reacional compatível aos resultados experimentais. Supondo um caminho de reação onde A adsorve associativamente ao sítio, e reage com $\mathrm{B}$ na fase gasosa, gerando um produto $\mathrm{C}$, chega-se a taxa de reação representada pela Equação 14:

$$
r=\frac{K_{R} K_{A} P_{A} P_{B}}{\left(1+K_{A} P_{A}+K_{C} P_{C}\right)}
$$

\subsection{Resolução com o MMQ}

Linearizando a Equação 14 chega-se na Equação 15, simplificada na Equação 16. 


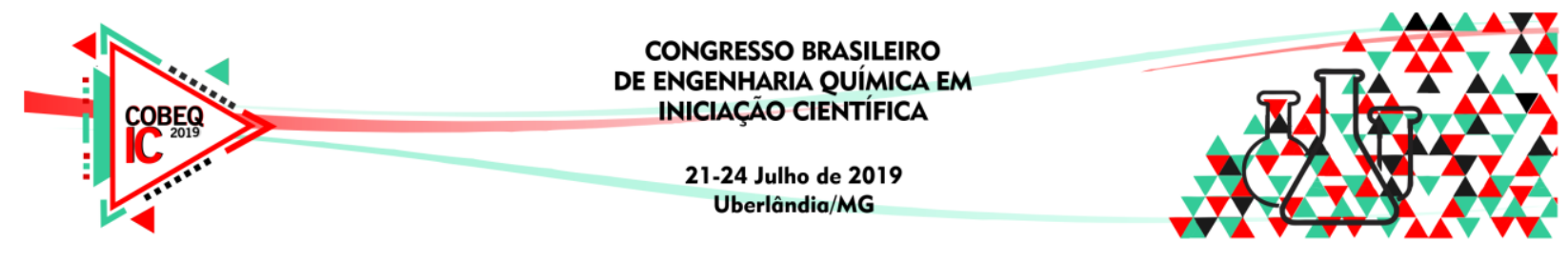

$$
\begin{aligned}
& \frac{P_{A} P_{B}}{r}=\frac{1}{K_{R} K_{A}}+\frac{1}{K_{R}} P_{A}+\frac{K_{C}}{K_{R} K_{A}} P_{C} \\
& y=\beta+\alpha_{1} x_{1}+\alpha_{2} x_{2}
\end{aligned}
$$

A Tabela 2 deve então ser construída.

Tabela 2 - Dados para a equação linearizada

\begin{tabular}{|c|c|c|}
\hline$\frac{P_{A} P_{B}}{r}$ & $\mathrm{P}_{\mathrm{A}}(\mathrm{atm})$ & $\mathrm{P}_{\mathrm{C}}($ atm $)$ \\
\hline 1,370 & 0,1 & 1 \\
\hline 2,924 & 1 & 10 \\
\hline 18,52 & 10 & 1 \\
\hline 2,941 & 1 & 20 \\
\hline 6,944 & 1 & 20 \\
\hline 35,71 & 20 & 1 \\
\hline 2,941 & 1 & 1 \\
\hline 4,444 & 1 & 20 \\
\hline
\end{tabular}

Através do MMQ faz o seguinte: No $1^{\circ}$ ambiente insere-se o número de experimentos, que no caso são 8 . No $2^{\circ}$ ambiente insere-se o número de variáveis independentes, no caso $\mathrm{P}_{\mathrm{A}}$ e $P_{C}$, então 2. Indo para o $3^{\circ}$ ambiente deve-se inserir a Tabela 2 . Finalizando, no $4^{\circ}$ ambiente o programa fornece os resultados. Através da Figura 1 pode-se observar o passo a passo.

Figura 1 - Ambientes do programa MMQ na resolução do problema

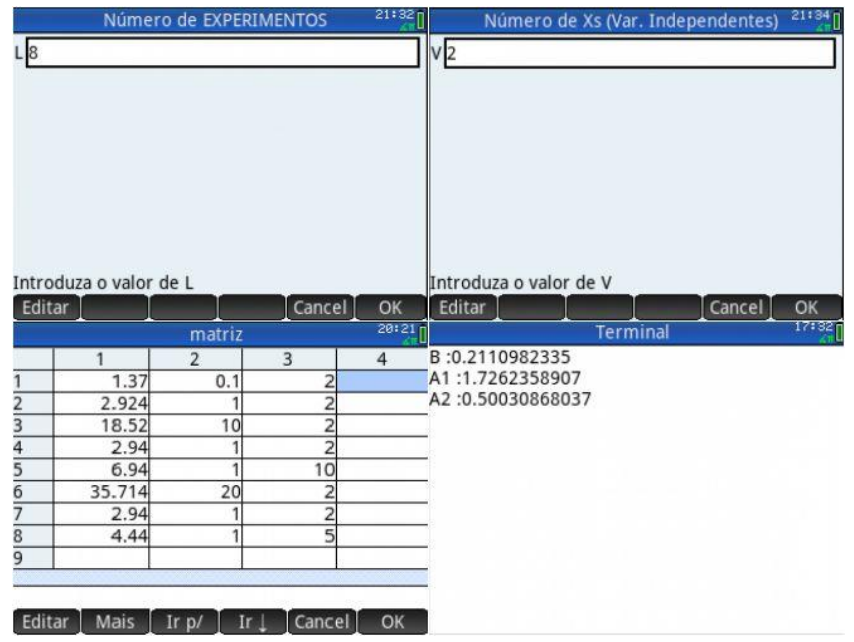

Resolvendo o sistema de equações formado pelo resultado das constantes, chega-se aos valores de $K_{R}=0,579$ g.(s.gcat.atm $)^{-1}, K_{A}=8,18 \mathrm{~atm}^{-1}, K_{C}=2,37 \mathrm{~atm}^{-1}$.

\subsection{Resolução com o Polymath}

Utilizando a regressão não linear através do Polymath, o resultado encontrado é o exibido na Figura 2. Observa-se um $\mathrm{R}^{2}$ igual a 0,9983 aproximadamente, o que indica uma compatibilidade entre o mecanismo proposto e os dados experimentais. 


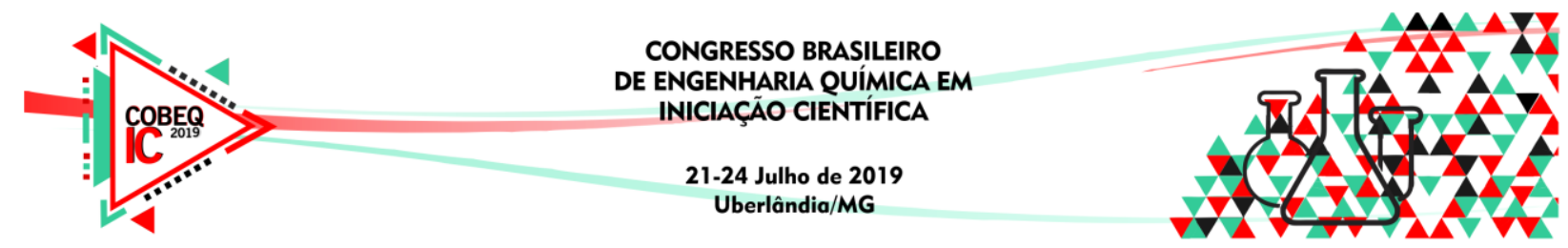

Figura 2 - Resultado da regressão não linear Polymath

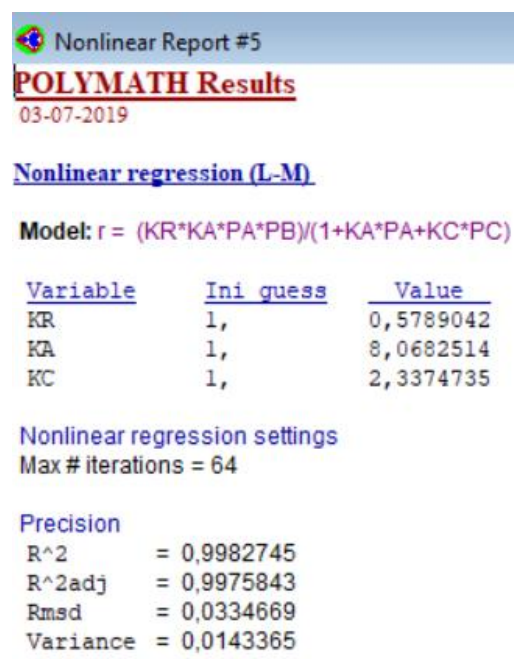

\subsection{Análise das respostas}

A regressão não linear é, de fato, uma análise mais exata e confiável que a regressão linear pelo método dos mínimos múltiplos quadrados, que deve servir apenas de parâmetro inicial, para o começo do processo iterativo aplicado no Polymath. Porém, nota-se através da Tabela 3, considerando 3 algarismos significativos, uma notável proximidade dos resultados, com erros acumulados relativamente pequenos.

Tabela 3 - Erros para cada constante encontrada

\begin{tabular}{|c|c|c|c|}
\hline Constante & $\begin{array}{c}\text { Resultado } \\
\text { Regressão } \\
\text { Não Linear }\end{array}$ & $\begin{array}{c}\text { Resultado } \\
\text { Regressão Linear } \\
\text { Múltipla }\end{array}$ & Erro Porcentual \\
\hline \hline $\mathrm{K}_{\mathrm{R}}$ (s.gcat.atm) & 0,579 & 0,579 & $0 \%$ \\
\hline $\mathrm{K}_{\mathrm{A}}\left(\mathrm{atm}^{-1}\right)$ & 8,07 & 8,18 & $0,0136 \%$ \\
\hline $\mathrm{K}_{\mathrm{C}}\left(\mathrm{atm}^{-1}\right)$ & 2,34 & 2,37 & $0,0128 \%$ \\
\hline
\end{tabular}

\section{REFERÊNCIAS}

ALMEIDA, R. N. de. O Método dos Mínimos Quadrados: Estudo e Aplicações para o Ensino Médio. Campos dos Goytacazes: UENF, 2015.

FOGLER, H. S. Elementos de Engenharia das Reações Químicas. Rio de Janeiro: LTC, 2009.

MAIA, A. da S. Catálise Heterogênea: Princípios. João Pessoa, 2010. Disponível em: encurtador.com.br/dlzDX. Acessado em: 19 de março de 2019.

SCHMAL, M. Cinética e Reatores: Aplicação na Engenharia Química; Teoria e Exercícios. Rio de Janeiro: Synergia, 2010. 